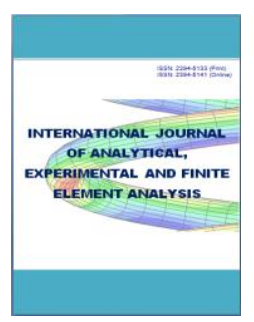

Mehmet ÇINAR

engmcinar@gmail.com

Bitlis Eren University

Bitlis / TURKEY

\title{
Finite Element Analysis and Drawing of Magnetic Flux Path with the Developed Program
}

Abstract - One of the methods used in the solution of partial differential equations is the finite element method. The solution region of the differential equation to be solved in finite element method is divided into sub-sections. When making finite element analysis, magnetic flux path drawing is made by making use of vector potential values of the nodes in the solution of the magnetic region. Thus, the finite element analysis gives information about the magnetic structure of the region. However, it is useful to use the moving finite element method instead of the classical finite element method when time dependent partial differential equations change and the solution network changes regionally.

In this article, drawing of magnetic flux path used in finite element analysis is mentioned. Application of a $\mathrm{C}++$ based software has been realized and the sample magnetic flux path drawings have been obtained.

Index Terms: Finite element analysis, magnetic flux path, partial differential equation

\section{INTRODUCTION}

In order to solve a large number of problems in engineering, physical sciences, social sciences and many other disciplines, it is necessary to formulate these problems with mathematical expressions, and then to find out some boundary conditions and the functions that make up the solutions using the initial conditions. These mathematical expressions that formulate a known problem sometimes include at least first order or higher order derivatives of the function sought. This kind of mathematical expression is called differential equation.

If the number of independent variables in a differential equation is two or more and the equation contains at least one partial derivative of any order, this equation is called "Partial Differential Equations [1]. Such equations are used in many engineering disciplines. In general, a partial differential equation including $x, y$ independent variables and $u(x, y \ldots)$ connected variables: $F\left(x, y, \ldots u, u_{x}, u_{y}, u_{x x}, u_{x y}, u_{y y}, \ldots\right)=0$ is written in the form. Here are partial derivatives of the unknown function with respect to the independent variables, and each of them is:

$$
\begin{aligned}
& u_{x}=\frac{\partial u}{\partial x}, u_{y}=\frac{\partial u}{\partial y}, u_{x x}=\frac{\partial^{2} u}{\partial x^{2}}, \\
& u_{x y}=\frac{\partial^{2} u}{\partial y \partial x}, \ldots
\end{aligned}
$$

is expressed as [2].

The highest order derivative in a partial differential equation is called the order of the differential equation. The equation is linear if the unknown function $u$ and its partial derivatives are first order. Generally, the second order partial differential equation with two independent variables is expressed as follows.

$$
\begin{aligned}
& A(x, y) \frac{\partial^{2} u}{\partial x^{2}}+B(x, y) \frac{\partial^{2} u}{\partial x \partial y}+C(x, y) \frac{\partial^{2} u}{\partial y^{2}}+ \\
& D(x, y) \frac{\partial u}{\partial x}+E(x, y) \frac{\partial u}{\partial y}+F(x, y) u=G(x, y)
\end{aligned}
$$

If the coefficients of the unknown function $u=u(x, y)$ and their partial derivatives are constant numbers, the 
differential equation with constant coefficients is obtained. Such an equation:

$$
\begin{aligned}
& A \frac{\partial^{2} u}{\partial x^{2}}+B \frac{\partial^{2} u}{\partial x \partial y}+C \frac{\partial^{2} u}{\partial y^{2}}+D \frac{\partial u}{\partial x}+ \\
& E \frac{\partial u}{\partial y}+F u=G(x, y)
\end{aligned}
$$

is written.

Second order partial derivatives play an important role in the classification of the differential equation. According to the sign of the $B^{2}-4 A C$ discriminant value, the above equation is classified as hyperbolic, parabolic and elliptic type. Accordingly, the above differential equation:

$B^{2}-4 A C>0$ is hyperbolic,

$B^{2}-4 A C=0$ is parabolic

$B^{2}-4 A C<0$ Elliptical

type differential equation is called [3].

Heat, wave and Laplace (potential) equations, which are the most important equations of mathematics physics, can also be classified as above. One-dimensional wave equation including $x$ position, $t$ time variable:

$\frac{\partial^{2} u}{\partial t^{2}}=\beta \frac{\partial^{2} u}{\partial x^{2}} \quad$ hyperbolic type

One dimensional heat equation:

$\frac{\partial u}{\partial t}=\alpha \frac{\partial^{2} u}{\partial x^{2}} \quad$ parabolic type

Two-dimensional Laplace equation for the function $u(x$, $y$ ) including $x$ and $y$ position variables:

$$
\frac{\partial^{2} u}{\partial x^{2}}+\frac{\partial^{2} u}{\partial y^{2}}=0
$$

and Poisson equation

$$
\frac{\partial^{2} u}{\partial x^{2}}+\frac{\partial^{2} u}{\partial y^{2}}=f(x, y)
$$

elliptic equations [4].
One of the most important issues in the solution of partial differential equations is the adaptive production of the solution network or grid. Variational approach is widely used for solution network generation and adaptation in two dimensional spatial situation. The variational approach is part of mathematical analysis. It is applied to mathematical and physical problems and to solve many engineering problems [5].

\section{Magnetic FluX Path Drawing}

The flux path of this magnetic structure can be drawn by using the vector potential values of the nodes in the solution of a magnetic region with finite elements or finite differences. Drawing the flux path gives important information about the magnetic structure. However, the variation of the vector potential value by region is more easily observed on this scheme. This amount of change gives the idea of which region should be divided into more triangular elements when dividing the solution region into triangular elements. In order to increase the accuracy of the calculated results, the regions where the vector potential change is high are divided into smaller triangular elements [6].

$B=\operatorname{Rot} A$

There is a relationship between vector potential and magnetic induction in equation (7). As it can be understood from this statement, when the equipotential points in the solution region are found and mapped, this map is also the flux path diagram. If the vector potentials of the corner nodes of a triangular element are known, the potential equation in the desired coordinate can be found as in equation (8) using the test function valid at all points in the triangle or on the triangle edges.

$A(x, y)=\alpha_{0}+\alpha_{1} x+\alpha_{2} y$

When drawing the equipotential curves, instead of calculating the vector potential value of a point on the triangle, there are points equal to a predetermined vector potential value to be mapped on the triangular element. For this, the vector potential value to be plotted is 
predetermined. The vector potential values to be plotted are drawn in two ways:

Starting from the smallest vector potential value and taking a certain amount of increments to draw the equivalent potential values. In this case, the total number of map lines is the ratio of the difference between the maximum vector potential value and the smallest vector potential value to the incremental amount.

After determining the total number of maps, the increment amount is obtained by dividing the difference between the largest vector potential value and the smallest value by the total number of lines. Starting from the smallest vector potential value, the same vector potential curves are drawn by increasing the amount of increments each time [7].

Drawings start from the smallest vector potential value. For the three sides of each triangular element, the value of the nodes of these edges is compared to the vector potential value plotted. It is searched whether the vector potential value is equal to the value of one of these two nodes or if there is a value between the vector potentials of these two nodes. If the vector being plotted is a value between two nodes of a triangular edge, the value on this edge must be equal to the mapped vector potential value and the vector potential value. The coordinates of this point are calculated from the coordinate and vector potential value of the edge nodes.

The vector potential value of $\mathrm{A}_{1}$ mapped as shown in Fig 1 is equal to $\mathrm{A}_{2}$ if the value between $\mathrm{A}_{1}$ and $\mathrm{A}_{2}$ is a value between $A_{1}$ and $A_{2}$. Since the vector potential value changes in a triangle element and at the sides are linear, for the $\mathrm{A}_{1}-\mathrm{A}_{2}$ edge;

$$
\frac{x_{2}-x_{L}}{x_{2}-x_{1}}=\frac{A_{2}-A_{L}}{A_{2}-A_{!}}
$$

can be written. Since all values except $X_{L}$ are known in this expression;

$$
\begin{aligned}
& x_{L}=\frac{\left(A_{1}-A_{2}\right) \cdot\left(x_{1}-x_{2}\right)-\left(A_{1}-A_{2}\right) \cdot x_{1}}{A_{2}-A_{1}} \\
& y_{L}=\frac{\left(A_{1}-A_{2}\right) \cdot\left(y_{1}-y_{2}\right)-\left(A_{1}-A_{2}\right) \cdot y_{1}}{A_{2}-A_{1}}
\end{aligned}
$$

The vector potential value of the coordinate $\left(X_{L}, Y_{L}\right)$ is equal to the vector potential value plotted on the map.

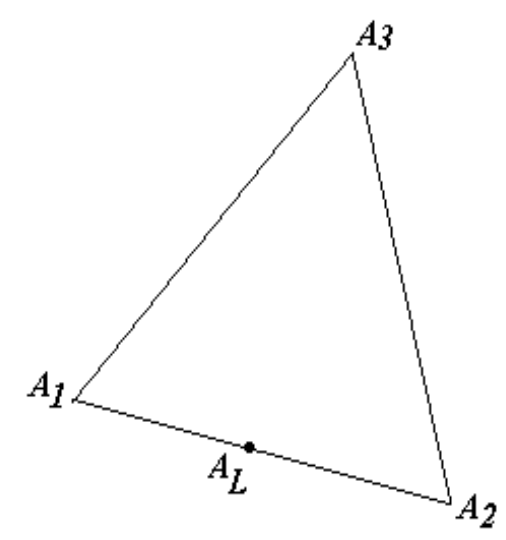

Fig. 1 Vector potential of $A_{L}$ point.

If there is a note equal to the vector potential value mapped on one side of a triangle, there is necessarily a point on one of the other two sides of this triangle whose vector potential is mapped. The line that connects this second point with the first point is the co-vector potential line.

For each of the increment values, the node vector potential values of all triangles are checked each time to obtain the identical vector potential curves of the entire solution region with the plotted potential lines.

Fig. 2 shows the flow diagram of the developed algorithm. 


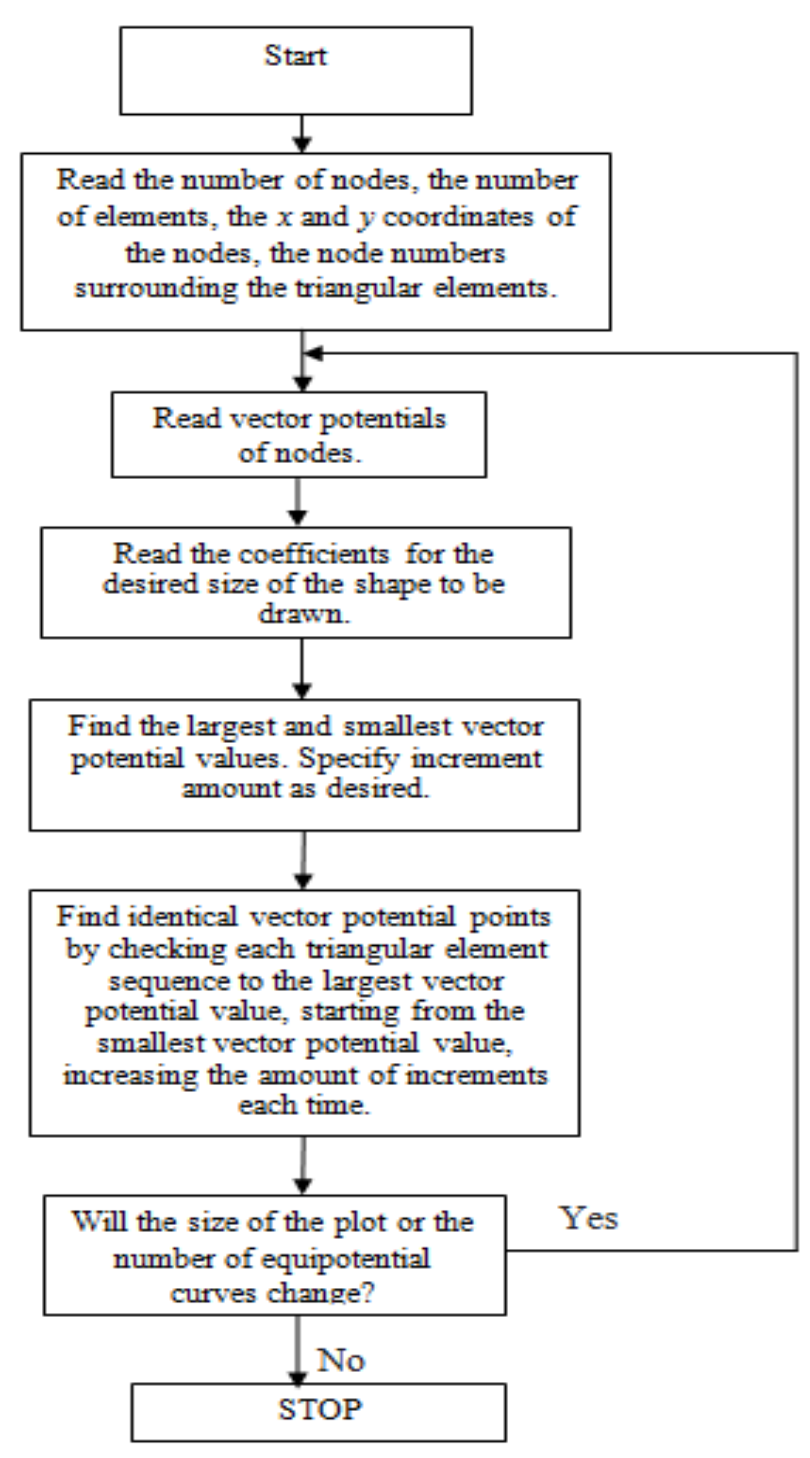

Fig. 2 Algorithm flow chart

\section{EXAMPLES}

a) Magnetic flux path curves (15 curves) of transformer analyzed by finite element method using monitor function $\left(u(x, y)=0.5\left(y-9.0-4 \sin \frac{2 \pi x}{21}\right)\right.$

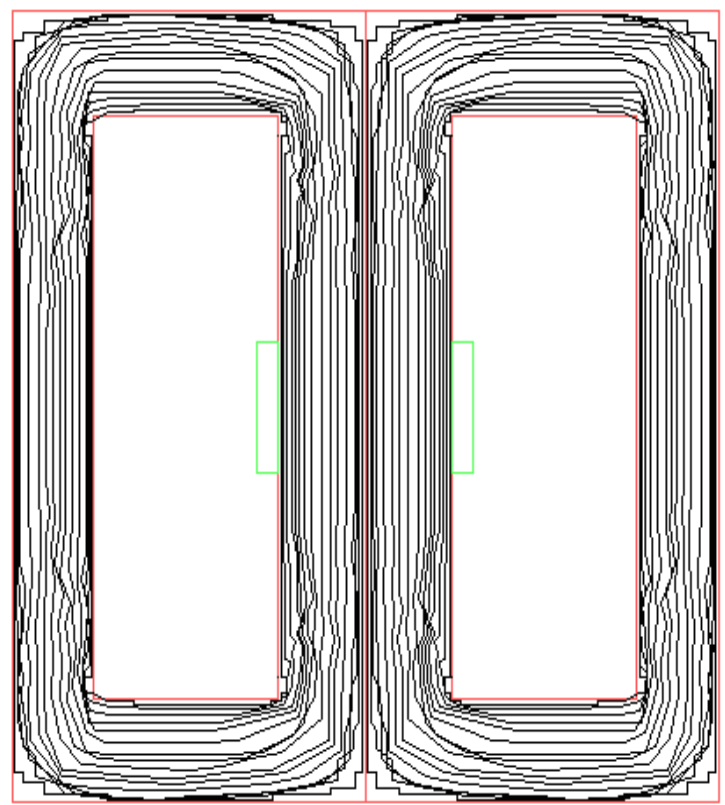

Fig. 3 Magnetic flux path curves of the transformer (15 curves)

b) Magnetic flux path curves (45 curves) of transformer analyzed by finite element method using monitor function $\left(u(x, y)=0.5\left(y-9.0-4 \sin \frac{2 \pi x}{21}\right)\right.$

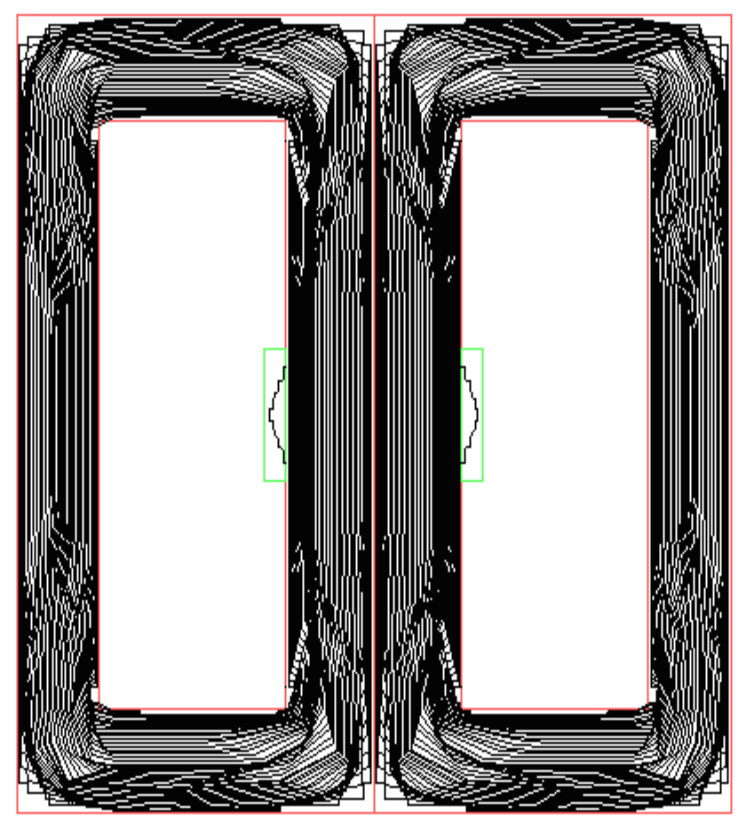

Fig. 4 Magnetic flux path curves of the transformer (45 curves) 
c) Magnetic flux path curves (100 curves) of transformer analyzed by finite element method using monitor function $\left(u(x, y)=0.5\left(y-9.0-4 \sin \frac{2 \pi x}{21}\right)\right.$

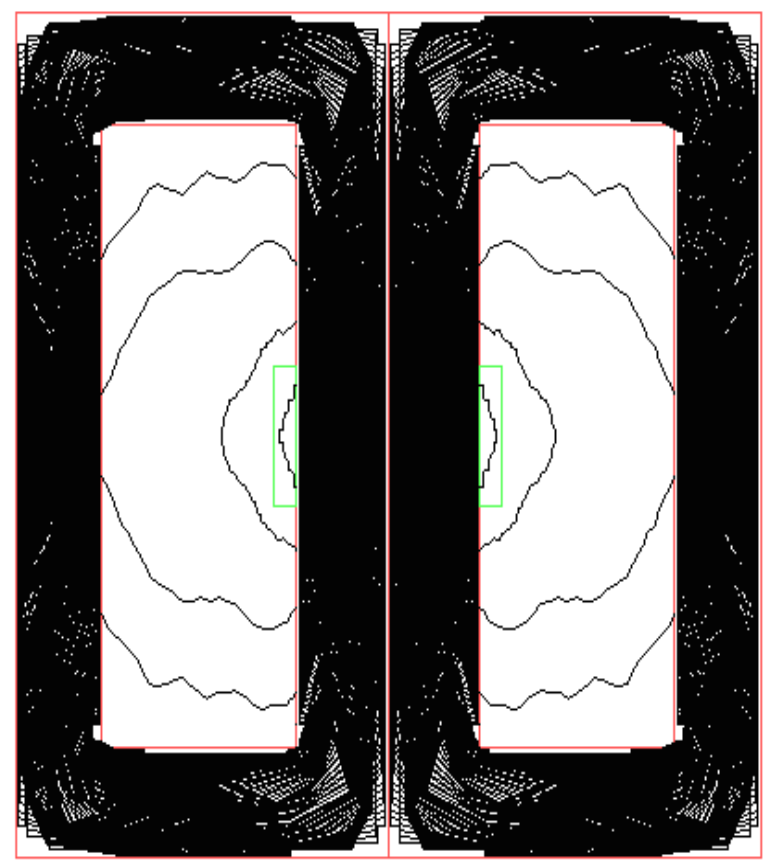

Fig. 5 Magnetic flux path curves of the transformer (100 curves

\section{RESULTS}

The most important issue in finite element analysis is the subdivision of the solution network. Especially when the magnetic flux path curves are drawn during the analysis, it contains important information in the solution stage. In areas where magnetic flux density is high, the curves are plotted more so that the solution is closer to the actual values.

\section{REFERENCES}

[1] Mehmet Aydın, Beno Kuryel, Gönül Gündüz, Galip Oturanç, 2001,” Diferansiyel Denklemler ve Uygulamalart",İzmir.

[2] R. Rannacher, 2001, "Adaptive Galerkin Finite Element Methods for Partial Differential Equations", Journal of Computational and Applied Mathematics, 128, 205-233.

[3] S.H. Lo., 2002, "Finite element mesh generation and adaptive meshing“, Prog. Struct. Analysis Materials, Vol:4, pp:381-399.

[4] Baker TJ. 1989, "Automatic mesh generation for complex three-dimensional regions using a constrained Delaunay triangulation”, Engineering with Computers 5: 161-175.

[5] Lee CK., 2000, "Automatic metric advancing front triangulation over curved surfaces", Engineering Computations 17(1): 48-74.

[6] Shephard MS \& Georges MK. 1991, "Automatic threedimensional mesh generation by the finite octree technique", International Journal for Numerical Methods in Engineering 32: 709-749.

[7] Luiz Vello, Denis Zorin 2001, “4-8 Subdivision”, Computer Aided Geometric Design, vol:18, pp:397-427. 